\title{
Xenobiotic Nucleic Acid (XNA) Synthesis by Phi29 DNA Polymerase
}

\author{
Leticia L. Torres ${ }^{1}$ and Vitor B. Pinheiro ${ }^{1,2}$ \\ ${ }^{1}$ Department of Structural and Molecular Biology, University College London, London, \\ United Kingdom \\ ${ }^{2}$ Institute of Structural and Molecular Biology, Department of Biological Sciences, \\ Birkbeck, University of London, London, United Kingdom
}

Phi29 DNA polymerase (DNAP) is the replicative enzyme of the Bacillus subtilis bacteriophage Phi29. Its extraordinary processivity and its ability to perform isothermal amplification of DNA are central to many molecular biology applications, including high-sensitivity detection and large-scale production of DNA. We present here Phi29 DNAP as an efficient catalyst for the production of various artificial nucleic acids (XNAs) carrying backbone modifications such as 1,5-anhydrohexitol nucleic acid (HNA), 2'-deoxy-2'-fluoro-arabinonucleic acid (FANA), and $2^{\prime}$-fluoro- $2^{\prime}$-deoxyribonucleic acid (2'-fluoro-DNA). A full protocol for the synthesis of HNA polymers by an exonuclease-deficient variant (D12A) of Phi29 DNAP plus a detailed guide for the design and test of novel XNA synthetase reactions performed by Phi29 DNAP are provided. (C) 2018 by John Wiley \& Sons, Inc.

Keywords: HNA • Phi29 DNA polymerase • XNA synthesis

How to cite this article:

Torres, L. L., \& Pinheiro, V. B. (2018). Xenobiotic nucleic acid (XNA) synthesis by Phi29 DNA polymerase. Current Protocols in Chemical Biology, 10, e41. doi: 10.1002/cpch.41

\section{INTRODUCTION}

Xenobiotic nucleic acids (XNAs) differ from their natural counterparts in at least one constituent chemical moiety: Phosphate, sugar, or nucleobase (Pinheiro \& Holliger, 2014). Backbone-modified XNAs (i.e., that differ on the phosphate or sugar) are not generally accepted by natural DNA polymerases (DNAPs), requiring them to be engineered. Engineering of XNA synthetases (DNA $\rightarrow$ XNA) has been most successful (with regard to the number of accessible XNA chemistries) with thermostable B-family DNAPs (Cozens, Pinheiro, Vaisman, Woodgate, \& Holliger, 2012; Di Pasquale et al., 2008; Dunn, Otto, Fenton, \& Chaput, 2016; Pinheiro, Loakes, \& Holliger, 2012; Ramsay et al., 2010), but significant advances have also been reported in A-family DNAPs (Chen et al., 2016; Ghadessy et al., 2004) and monomeric RNA polymerases (Ibach et al., 2013; Sousa \& Padilla, 1995).

Phi29 DNAP is the replicative polymerase of the Bacillus subtilis bacteriophage Phi29. It is presented here for the first time as a mesophilic alternative for the synthesis of XNAs. Phi29 DNAP is a well-characterized enzyme (Salas, Holguera, Redrejo-Rodríguez, \& de Vega, 2016) with considerable potential in biotechnology because of its extreme processivity (Blanco, Bernads, Lharo, Martins, \& Garmendia, 1989), high fidelity, and strand-displacing activity, which enable the isothermal amplification and large-scale synthesis of DNA (Dean, Nelson, Giesler, \& Lasken, 2001). 
In addition, the high processivity of Phi29 DNAP also facilitates the engineering of novel XNA polymerases. Engineering of an archaeal DNA polymerase from Thermococcus gorgonarius for the synthesis of RNA (Cozens et al., 2012) highlighted that a major contributor to RNA polymerase function was high affinity for the primer/template nascent duplex, which is also a key factor in enzyme processivity.

Like the engineered DNAPs of T. gorgonarius (Pinheiro et al., 2012) and Thermococcus kodakarensis (Dunn et al., 2016), Phi29 DNAP is a B-family polymerase (Mönttinen, Ravantti, Stuart, \& Poranen, 2014). Phi29 DNAP is also part of a subset of polymerases that are naturally primed by a protein (instead of a nucleic acid oligomer) and are particularly involved in the replication of chromosomes or plasmids of linear topology (Salas, 1991). In fact, Phi29 DNAP is an exception among protein-primed polymerases because it is able to initiate DNA synthesis not only from a protein, but also from DNA and RNA primer oligonucleotides (Blanco \& Salas, 1996).

The high processivity and strand-displacing activities of Phi29 have been mapped to a Phi29-specific domain, terminal protein region 2 (TPR2; Rodríguez et al., 2005) that, along with palm, fingers, and thumb subdomains, form an internal ring structure that clamps tightly around the DNA nascent duplex, improving the polymerase binding to DNA and thus increasing the residence time on the replicating molecule. The remarkable processivity of Phi29 DNAP is assumed to have a positive impact on the synthesis of XNA polymers. While the number of amino acids effectively contacting oligomers of alternative chemistries can diminish, the natural clamping strategy of Phi29 DNAP to the double-stranded DNA (dsDNA) provides a mechanical contribution to the binding of novel heteroduplexes (DNA:XNA), that potentially translates into longer stretches of XNA being synthesized.

An exonuclease-deficient Phi29 DNA polymerase (D12A, Bernad, Blanco, Lkaro, Martin, \& Salas, 1989; see Critical Parameters, Phi29 exonuclease activity section) is an efficient XNA synthase for 1,5-anhydrohexitol nucleic acid (HNA), 2'-deoxy-2'fluoro-arabinonucleic acid (FANA), and 2'-fluoro-DNA - chemistries that do not create significant steric clashes in the active site. Engineering Phi29 DNAP further improves XNA polymerase activity (L. L. Torres and V. B. Pinheiro, unpub. data), but synthesis conditions do not significantly differ from the protocol presented here. In this protocol, we introduce Phi29 DNAP as a mesophilic alternative for the template-dependent primed synthesis of XNAs, explaining key points to consider when testing new XNA chemistries as substrates, and providing, as an example, a full protocol for the synthesis of HNA.

\section{STRATEGIC PLANNING}

While longer reaction times tend to yield better XNA synthesis, the strong exonuclease activity of Phi29 DNAP can become an issue in the reaction. Using the DNA primer template combination described below and Phi29 DNAP for DNA synthesis, we have noticed that full-length product is observed after as little as $5 \mathrm{~min}$ but the quality of the reaction (full-length extension as a fraction of the total product) improves with incubations up to $3 \mathrm{hr}$. Longer reactions tend to be dominated by template-independent synthesis and exonuclease activity and have reduced quality. As such, while XNA synthesis can be conveniently carried out overnight, we recommend a time course be generated for all polymerase variant and XNA combinations being tested.

In addition, the protocol presented here has been optimized for the synthesis of short XNA molecules $(<100$ bases). Longer products are possible but require further optimization of reaction conditions and primer-template combinations (discussed in the Commentary). 


\section{1,5-ANHYDROHEXITOL NUCLEIC ACID (HNA) SYNTHESIS BY PHi29 DNA POLYMERASE}

Template-dependent HNA synthesis consists of the extension of a fluorescently labeled primer pre-annealed to a chosen DNA template, using an exonuclease-deficient Phi29 DNAP (D12A) mutant. Synthesis is carried out in vitro at $30^{\circ} \mathrm{C}$ using chemically synthesized 1,5-anhydrohexitol nucleoside triphosphates (hNTPs; Lagoja, Marchand, Van Aerschot, \& Herdewijn, 2003).

Wild-type Phi29 DNAP can be commercially obtained from different suppliers (e.g., New England Biolabs), but exonuclease-deficient mutants are heterologously expressed in Escherichia coli and purified. Phi29 DNAP variants can be extensively purified (Lazaro, Blanco, \& Salas, 1995; Takahashi et al., 2014), however, in our experience, a simple and fast partial purification of Phi29 DNAP variants by adsorption chromatography using heparin resins (Lazaro et al., 1995, Xiong, Zhang, \& He, 2008) is enough; the resulting polymerase ( $\sim 40 \%$ to $60 \%$ of the purified fraction) is sufficiently active for robust and quantitative XNA synthesis and free from contaminants that may interfere in the reaction.

Phi29 DNAP's enzymatic rate for DNA synthesis on a linear template is $2280 \mathrm{nt} / \mathrm{min}$ at $30^{\circ} \mathrm{C}$ (Soengas, Gutiérrez, \& Salas, 1995), but hNTP incorporation is significantly less efficient. The synthesis of a 56-mer HNA polymer can be completed with high efficiency in $1 \mathrm{hr}$, when using heparin-purified enzyme preparations. HNA can be purified after synthesis by denaturing gel electrophoresis, selective capture of HNA product, or by selective degradation of the DNA template.

\section{Materials}

Phi29 DNA polymerase D12A variant (heterologously expressed, heparin-purified)

Phi29 DNA polymerase $10 \times$ buffer (New England Biolabs; $50 \mathrm{mM}$ Tris. $\mathrm{Cl}$, $\mathrm{pH} 7.5 ; 10 \mathrm{mM} \mathrm{MgCl} 2 ; 10 \mathrm{mM}\left(\mathrm{NH}_{4}\right)_{2} \mathrm{SO}_{4} ; 4 \mathrm{mM}$ DTT; adjust to $\mathrm{pH} 7.5$ at $\left.25^{\circ} \mathrm{C}\right)$

$10 \mathrm{mg} / \mathrm{ml}$ ultrapure BSA

hNTPs, pre-mixed at $10 \mathrm{mM}$ (containing $2.5 \mathrm{mM}$ of each hATP, hCTP, hGTP, and hTTP; see Lagoja et al., 2003)

Primer (DNA, RNA, or 2'-O-methyl DNA, e.g., 5'-/56FAM/ CGGATCCGTTTAAGCTAGG-3')

DNA template, e.g., 5'-TGGTCCAGCATCGTGAGATCGATTACCGAACAGCAC TACGTGGCTAAGTGCTTATCTCCTAGCTTAAACGGATCCG-3'

$5 \mathrm{M}$ betaine

500 mM EDTA, pH 8.0

Thermal cycler or heat block (suitable for incubation of small volumes) 0.2-ml microcentrifuge tubes

1. Assemble primer-template annealing reactions in $0.2-\mathrm{ml}$ microcentrifuge tubes.

$\begin{array}{lll}\text { Typical } 20 \mu \mathrm{l} \text { reactions: } & \text { XNA synthesis } & \text { Primer only } \\ \text { NEB } 10 \times \text { Phi29 DNAP buffer } & 2 \mu \mathrm{l} & 2 \mu \mathrm{l} \\ 5 \mathrm{M} \text { betaine } & 4 \mu \mathrm{l} & 4 \mu \mathrm{l} \\ 10 \mu \mathrm{M} \text { DNA primer } & 0.2 \mu \mathrm{l} & 0.2 \mu \mathrm{l} \\ 10 \mu \mathrm{M} \text { DNA template } & 0.6 \mu \mathrm{l} & - \\ \mathrm{ddH}_{2} \mathrm{O} & 12.1 \mu \mathrm{l} & 12.7 \mu \mathrm{l}\end{array}$

XNA synthesis is carried out as a primer extension reaction. As such, primer-template annealing is an important consideration in the reaction. In our experience, pre-annealing is not always necessary: It is dependent on the primer-template combination and on the expected secondary structure of the DNA template being used. 
We describe the preparation of a 20- $\mu$ l reaction, which can be scaled up. Reactions of up to $100 \mu \mathrm{l}$ can be carried out in 0.2-ml microcentrifuge tubes, but we split larger reactions into $100-\mu l$ aliquots to minimize potential temperature and heat transfer variations. Smaller volume reactions are also possible (e.g., down to $10 \mu \mathrm{l}$ ), but they are more challenging to prepare, and more prone to suffer from volume reduction by evaporation in longer incubations.

Primer concentrations as well as primer/template molar ratios can influence the outcome of the reaction. A primer/template ratio of 1:3 (or higher template) yields good primer extension results, at the expense of not every template being extended, which can be a consideration in the isolation of aptamers.

A primer-only negative control is set up to monitor primer degradation, templateindependent synthesis, and formation of primer-protein complexes, which can lead to spurious fluorescent signals (see Troubleshooting section).

2. Anneal primer and template in thermal cycler using a denaturing step: $2 \mathrm{~min}, 95^{\circ} \mathrm{C}$ and an annealing ramp: $95^{\circ}$ to $18^{\circ} \mathrm{C}$ at a rate of $0.2^{\circ} \mathrm{C} / \mathrm{sec}$.

In our experience, based on multiple primer and template combinations, annealing is a robust process and outcome is not affected by small changes in the experimental parameters.

3. Add BSA, nucleotide triphosphates, and DNA polymerase to the reactions:

\section{$0.2 \mu \mathrm{l} \mathrm{BSA}(10 \mathrm{mg} / \mathrm{ml})$ \\ $0.4 \mu \mathrm{l}$ hTPs $(10 \mathrm{mM}$ mix $)$ \\ $0.5 \mu l$ Phi29 D12A DNAP $(1 \mu \mathrm{g} / \mathrm{ml})$}

As BSA and DNAP are not thermostable, they are added after primer-template annealing. We typically add XNA triphosphates (i.e., hNTPs for HNA synthesis) together with BSA and DNAP after primer annealing. While annealing conditions are not expected to degrade hNTPs, other chemistries (e.g., GNA) may have labile triphosphates that may degrade in those conditions. Therefore, we recommend as good practice to add xenobiotic nucleoside triphosphates $(x N T P S)$ at this step, since they are a limiting and expensive resource.

Reactions are carried out with $0.5 \mathrm{ng}$ DNAP. In comparing different XNA chemistries and different polymerase mutants, we find that the absolute amount of DNAP per reaction is more informative than polymerase activity (a measure of DNA synthesis against a DNA template) in normalizing different enzymes. The amount of DNAP in a particular enzyme preparation can be determined by SDS-PAGE using a BSA calibration curve.

4. Incubate reactions $1 \mathrm{hr}$ at $30^{\circ} \mathrm{C}$ in a thermal cycler.

Different reaction times are possible (and may be necessary) depending on the polymerase mutations and XNA chemistry being used.

5. Add $0.5 \mu 1500 \mathrm{mM}$ EDTA, $\mathrm{pH} 8.0$, to each reaction to quench.

Reactions can also be quenched by heat denaturation (e.g., $20 \mathrm{~min}$ at $65^{\circ} \mathrm{C}$ ) or by the addition of a loading buffer (e.g., 98\% v/v formamide, $10 \mathrm{mM}$ EDTA, 0.1\% SDS) for denaturing gel electrophoresis.

As the primer is fluorescently labeled, reaction products can be separated by denaturing polyacrylamide gel electrophoresis (urea-PAGE) according to previously described protocols (Summer, Grämer, \& Dröge, 2009), and visualized using a gel imaging system (e.g., Typhoon FLA 9500). An example of the result of an HNA extension reaction can be observed in Figure 1. 


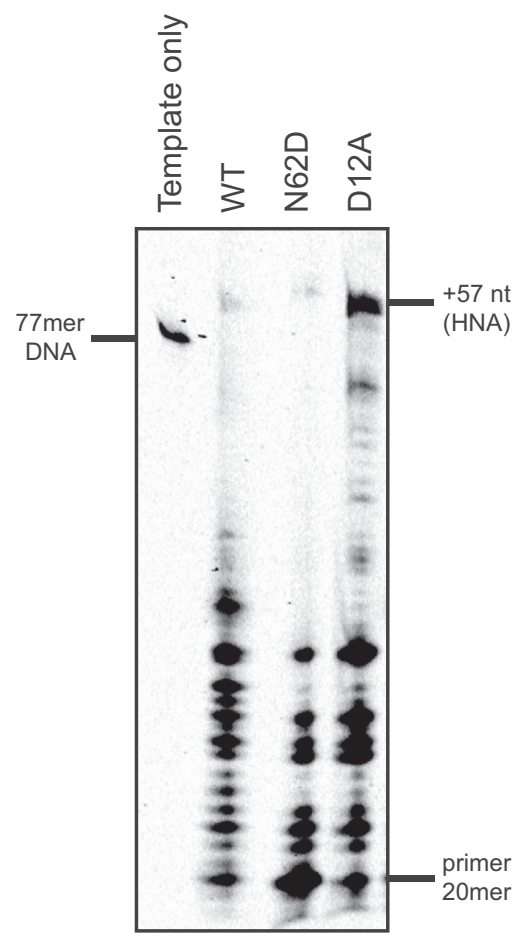

Figure 1 Efficient HNA synthesis by Phi29 DNAP D12A. HNA primer extension products synthesized by Phi29 DNAP wild type (WT; lane 2) and the two exonuclease-deficient variants, N62D (lane 3) and D12A (lane 4), were separated by denaturing PAGE and the products visualized by scanning the gel to detect fluorescently labeled primer. Incorporation of 57 nucleotides $(+57 \mathrm{nt})$ is the full extension possible in the given template. HNA migrates slower than DNA in a denaturing PAGE, as previously reported (Pinheiro et al., 2012).

\section{1,5-ANHYDROHEXITOL NUCLEIC ACID (HNA) PURIFICATION}

A successful HNA synthesis can be scaled up to produce larger amounts of HNA that can subsequently be purified from the remaining components of the synthesis reaction. While $20 \mu \mathrm{l}$ is the smallest recommended volume to perform diagnostic XNA synthesis reactions, purification of XNAs needs to start from a larger reaction volume of at least $100 \mu 1$.

Because HNA is resistant to nucleases (Pinheiro et al., 2012), the simplest route to purification is to selectively degrade the DNA primer and template by incubating the synthesis reaction with DNase. Afterwards, HNA polymers can be isolated by phenol/chloroform extraction and purified by precipitation in ethanol or isopropanol. Importantly, after a synthesis reaction Phi29 DNAP remains attached to its products of extension. Thus, while silica spin columns can also provide purification of HNA molecules, a phenol/chloroform extraction step is necessary to denature and disassemble the DNAP from the HNA polymer to be purified.

Alternatively, if full-length HNA needs to be isolated from intermediate products of extension, or in cases were the XNA synthesis efficiency is not optimum, which may be the result of multiple stalling events during synthesis or more challenging XNA chemistries, it is best to isolate the XNA product of interest from a denaturing polyacrylamide gel (Ellington \& Pollard, 2001). 


\section{Materials}

TURBO $^{\mathrm{TM}}$ DNase (Ambion)

25:24:1 phenol/chloroform/isoamyl alcohol saturated with $10 \mathrm{mM}$ Tris. $\mathrm{Cl}, \mathrm{pH} 8.0$

$3 \mathrm{M}$ sodium acetate $(\mathrm{NaOAc}), \mathrm{pH} 5.2$

Isopropanol, absolute

$70 \%(\mathrm{v} / \mathrm{v})$ ethanol

\section{5-ml microcentrifuge tubes}

Thermal cycler or heat block (suitable for incubation of small volumes)

Microcentrifuge

Vortex shaker

Spectrophotometer

1. Starting from a $100 \mu 1 \mathrm{HNA}$ synthesis (single $100 \mu \mathrm{l}$ reaction), add 2 units TURBO ${ }^{\mathrm{TM}}$ DNase to the reaction mix and incubate $1 \mathrm{hr}$ at $37^{\circ} \mathrm{C}$ in a thermal cycler.

Smaller volumes are difficult to handle and may result in a significant loss of yield from the purification. In addition, it is more difficult to detect purified HNA from smaller syntheses and more specialized equipment may be required [e.g., GE Typhoon FLA9500 if using fluorescein amidite (FAM)-labeled 2'-O-methyl-RNA primers]. In our experience TURBO ${ }^{T M}$ DNase presents the highest efficiency of DNA degradation in DNA:HNA mixes. Nevertheless, other nucleases can be used, e.g., DNase I, BAL-31 DNase.

2. Transfer reaction volume to a 1.5-ml microcentrifuge tube, add 1 volume of 25:24:1 (v/v/v) phenol/chloroform/isoamyl alcohol solution and vortex briefly.

Increasing the volume size of the completed reaction (to at least $200 \mu$ l) by diluting it with $\mathrm{dd}_{2} \mathrm{O}$ water before adding the phenol/chloroform/isoamyl alcohol solution usually improves recovery of the HNA.

3. Centrifuge resulting emulsion $5 \min , 18,000 \times g$ at room temperature to allow the partition of mixture into an aqueous phase (containing HNA) and an organic phase.

Buffer conditions and $\mathrm{pH}$ can affect whether nucleic acids partition into the aqueous or organic phase. In our experience, HNA polymers behave like DNA, localizing to the aqueous phase in alkali $\mathrm{pH}$ and in buffers containing potassium ions.

4. Recover top aqueous layer containing HNA and transfer it to a new 1.5-ml microcentrifuge tube.

In general, one extraction is enough to remove proteins but the purity of the HNA fraction can be improved with additional extractions.

5. To precipitate HNA, first add 0.1 volume $3 \mathrm{M} \mathrm{NaOAc}, \mathrm{pH} 5.2$ and mix by pipetting up and down. Then add 2.5 volumes of absolute isopropanol and mix sample by inverting tube several times.

Precipitation by ammonium acetate (0.4 M final concentration) can also be performed but we observe better yields when using the sodium salt. Nucleic acid co-precipitants like glycogen can also be added to enhance HNA precipitation.

6. Incubate sample at $-20^{\circ} \mathrm{C}$ at least $1 \mathrm{hr}$.

Incubations at $4^{\circ} \mathrm{C}$ (or even at room temperature) and for longer times (overnight) can increase the precipitation yield, especially when expected HNA amounts are low.

7. Centrifuge sample at least $1 \mathrm{hr}, 18,000 \times g$, room temperature.

You should observe a white pellet at the bottom of the tube after the final centrifugation step. The length of the centrifugation is crucial for proper HNA recovery. Shorter times will significantly reduce the yield.

Torres and Pinheiro
8. Discard supernatant, taking special care to not lose HNA pellet if it becomes dislodged from the tube wall. 
9. To remove remaining salts, add $500 \mu 170 \%$ (v/v) ethanol solution and wash pellet by inverting tube up and down several times.

If the pellet becomes resuspended, microcentrifuge the sample again 5 min at $18,000 \times g$, room temperature.

10. Discard ethanol solution, drain excess by inverting tube upside down on a paper towel for $1 \mathrm{~min}$ and let sample air-dry $\sim 20 \mathrm{~min}$ at room temperature.

11. Resuspend HNA pellet into $10 \mu \mathrm{lddH_{2 }} \mathrm{O}$.

12. Determine concentration by measuring UV absorbance at $260 \mathrm{~nm}$.

Under some precipitation conditions (e.g., when using sodium acetate and high alcohol concentrations) a significant amount of free nucleotides (and short DNA molecules) can co-precipitate with HNA polymers and lead to overestimation of HNA concentration. Denaturing gel electrophoresis of an aliquot of the purified HNA in parallel with a DNA calibration curve (using a DNA fragment of the same size and sequence than HNA) can provide an additional, accurate estimate of recovery.

\section{COMMENTARY}

\section{Background Information}

The low chemical diversity and highly homogeneous composition of DNA (and RNA) that underpins its unique potential for genetic information storage has also led to the exquisite specialization of its replication machinery, i.e., polymerases.

The introduction of functional diversity at the nucleic acid backbone has proven to be of importance for the development of new ligands (aptamers, siRNA; Bramsen, Grünweller, Hartmann, \& Kjems, 2014; Ruckman et al., 1998), catalysts (XNAZymes; Taylor \& Holliger, 2015), and nanostructures with potential applications as new materials (XNA origami; Taylor et al., 2016). Indeed, XNAs can have physicochemical properties that make them highly relevant to biomedical and biotechnological applications: Higher resistance to nucleases, increased duplex stability (to DNA, RNA, or to the XNA itself), improved pharmacokinetic properties, and reduced immunogenic potential (Bramsen et al., 2014; Pinheiro \& Holliger, 2014).

In general, changes to any of the three chemical moieties of natural nucleic acids have a negative impact on polymerization through steric clashes, lack of stabilizing interactions, or through nascent duplex distortion. Nevertheless, it is possible to engineer polymerases with expanded substrate repertoires to allow the synthesis, and even evolution, of XNA polymers. For instance, variants of the Thermococcus aquaticus DNAP obtained by in vitro selection can generate amplification products of up to $2 \mathrm{~kb}$ of fully phosphorothioate-substituted DNA (Ghadessy et al., 2004), which is resistant to exonucle- ases and can be further chemically modified with iodoacetamides. A commercial version of the archaean $9^{\circ} \mathrm{N}$ DNAP (Therminator, New England BioLabs) carrying a single mutation (A485L; Gardner, Joyce, \& Jack, 2004) can copy DNA templates into threose nucleic acid (TNA) polymers with high fidelity (Ichida, Horhota, Zou, McLaughlin, \& Szostak, 2005), generating an XNA shown to be resistant to degradation in human serum (Culbertson et al., 2016).

In a more extensive work of engineering through directed evolution and rational design, the DNAP from T. gorgonarius has been engineered for the synthesis of eight different XNAs, including HNA, FANA, 2'-fluoro-DNA, TNA, cyclohexenyl nucleic acid (CeNA), locked nucleic acid (LNA), arabinonucleic acid (ANA), and 2'-azido-2deoxyribonucleic acid (Pinheiro et al., 2012). Extending the substrate spectrum of polymerases is not limited to DNAPs. The RNA polymerase from the bacteriophage $\mathrm{T} 7$ has been successfully engineered, for instance, for the efficient synthesis of 2'-O-methyl DNA (Ibach et al., 2013).

Phi29 DNAP is an enzyme with a remarkable potential for XNA research because of its extreme processivity and strand-displacing activity. The high processivity decreases the engineering challenge towards XNA synthesis, as demonstrated here with D12A Phi29 DNAP where a single mutation is sufficient to enable the synthesis of HNA (in contrast to the fourteen substitutions $T$. gorgonarius DNAP needed to develop the same activity (Pinheiro et al., 2012), FANA (not shown), or 2'-F DNA (not shown). Phi29 DNAP may also enable
Torres and Pinheiro 
other applications, such as XNA synthesis by rolling circle amplification and in vivo XNA applications.

\section{Critical Parameters}

\section{Phi29 exonuclease activity}

Wild-type Phi29 DNAP displays strong exonuclease activity, efficiently degrading both single- and double-stranded DNA substrates in the $3^{\prime}$ to $5^{\prime}$ direction. Such exonuclease activity is at the heart of the enzyme's proofreading ability and it improves its intrinsic insertion discrimination $\left(10^{-4}\right.$ to $\left.10^{-6}\right) 100$ fold (Esteban, Salas, \& Blanco, 1993; Garmendia, Bernad, Esteban, Blanco, \& Salas, 1992), resulting in a polymerase of low mutational rate that can ensure accurate extension products.

Proofreading activity is generally a desired feature of polymerases at the time of DNA replication, however, the incorporation of non-canonical nucleotides can be drastically impaired by the polymerase exonuclease activity (Figure 2). Presumably, the lower incorporation kinetics and the induced distortion on the nascent hybrid duplex lead to greater sampling of the exonuclease domain and repeated removal of incorporated XNA triphosphates. The most common solution to this problem has been to make use of an exonucleasedeficient mutant of the polymerase, either by mutating catalytic residues involved in the exonucleotlytic activity (Asp12, Glu14, Asp66, Asp169, and Tyr165; Beese \& Steitz, 1991; Bernad et al., 1989; Esteban, Soengas, Salas, \& Blanco, 1994) or targeting residues that shift the dynamics of the enzyme away from exonuclease activity (Thr15 and Asn62; Vega, Lazaro, Salas, \& Blanco, 1996).

In Phi29 DNAP, residues implicated in exonucleolysis also affect the polymerase strand-displacing activity (Esteban et al., 1994; Soengas et al., 1992), thus the D12A exonuclease-deficient enzyme used here is better suited for linear syntheses where strand displacement is not required. The second alternative, substitutions on Phi29 DNAP residues Thr 15 or Asn62 preferentially by negatively charged amino acids, produce enzymes with an altered capacity to bind single-stranded DNA: Less efficient at exonucleolysis (between $80 \%$ and $90 \%$ reduction), but still capable of amplifying double-stranded DNA substrates and performing strand displacement (Vega et al., 1996).

Interfering with the polymerase dynamics is an effective strategy to reduce exonuclease activity in DNA synthesis but, depending on the XNA being synthesized, it may remain too high for efficient XNA synthesis. That can be at least partly bypassed through the use of phosphorothioate-containing oligonucleotide primers that are exonuclease resistant.

\section{Primer design}

The $5^{\prime}$ end of the primer is usually modified to incorporate a label that can be either fluorescent or radioactive, and that will allow easy and sensitive visualization of the extension products.

The $3^{\prime}$ end of the primer is very sensitive to Phi29 DNAP $3^{\prime}$ to $5^{\prime}$ exonuclease activity. In order to protect the primer from degradation, the $3^{\prime}$ end can be modified by the incorporation of two phosphorothioate (PS) groups (where one of the non-bridging phosphate oxygens is replaced with a sulfur atom) in place of the canonical phosphate groups. The hydrolysis of PS bonds occurs at a substantially lower rate than that of the natural phosphodiester bonds, increasing the half-life of the oligonucleotide and hence the probability of the primer becoming extended. PS-primers are commercially available and can be ordered as 3 '-end modified DNA oligomers from most suppliers. Use of PS primers does not affect XNA synthesis or purification, which remain as described above.

If opting for mutations that do not abolish exonuclease activity completely in the synthesis of a nuclease-sensitive XNA, an initial optimization of the incubation time should be performed. Longer incubation times will usually increase product accumulation but will also expose the newly synthesized XNA to Phi29 DNAP $3^{\prime}$ to $5^{\prime}$ degradation for longer periods of time. Thus, a time course synthesis to find a window of visualization is suggested.

\section{Template design}

Polymerases incorporate xenobiotic nucleoside triphosphates (xNTPs) at different rates in different sequence contexts. For instance, Phi29 DNAP incorporates purines more easily than pyrimidine 1,5 -anhydrohexitol nucleoside triphosphates (hNTPs), and so an initial bias of the template sequence to purine incorporation favors the overall extension reaction. Depending on the XNA chemistry of choice and on the application of the synthesized XNA, different template sequences of similar length should be tested in order to find one that can be copied without major enzymatic stalling events, which could lead to incomplete extension. 


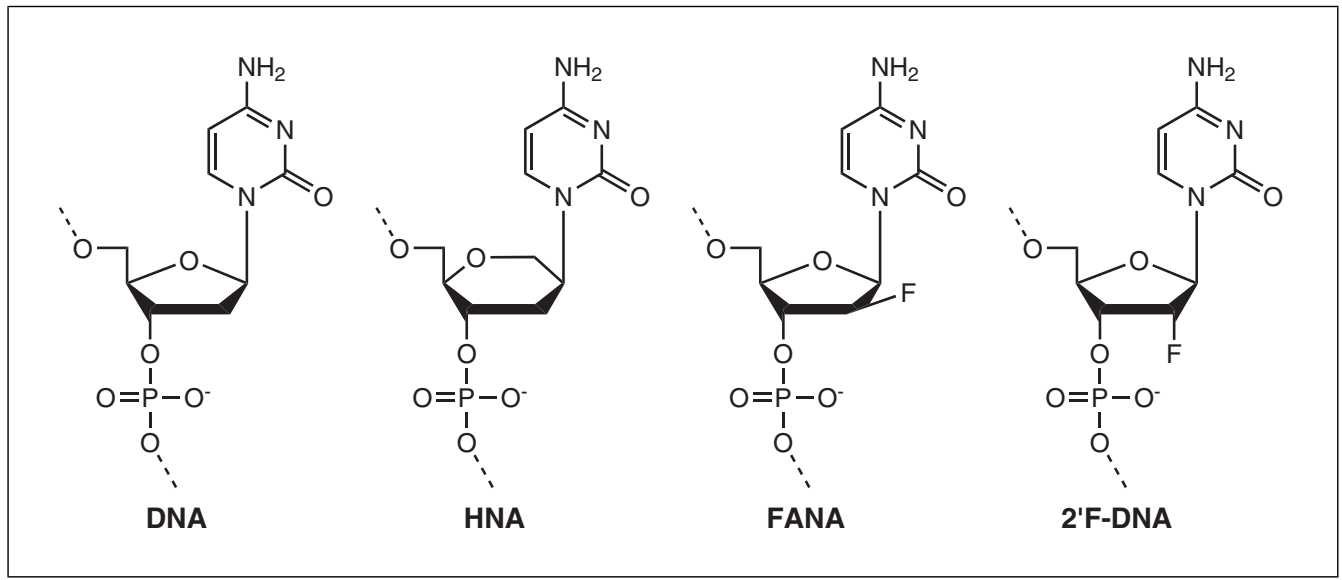

Figure 2 XNA chemistries accessible to Phi29 (D12A) DNAP variant. The D12A mutant of Phi29 DNAP remains an efficient DNA polymerase and it can be used in the efficient synthesis of 1,5-anhydrohexitol nucleic acid (HNA), 2'-deoxy-2'-fluoro-arabinonucleic acid (FANA), and 2'-fluoro-2'-deoxyribonucleic acid (2'F-DNA).

In some templates Phi29 DNAP can preferentially use the template itself as a primer performing extension from its $3^{\prime}$ end, which consumes the components of the reaction and reduces the yield of the desired extension product. Visualization of both template and primer post-synthesis using a denaturing PAGE is an efficient strategy to determine how much this side reaction interferes with the desired XNA synthesis. This can be achieved by using a fluorescently labeled template, e.g., Cy5 if synthesis primer is fluorescein amidite (FAM) labeled to ensure minimal fluorescent spectral overlap or using the experimental setup described above. In the latter case, primer extensions are identified by the FAM fluorescence (or primer fluorophore if different). Template extensions can then be visualized by staining the gel with high-affinity nucleic acid dyes such as SYBR Gold, which efficiently stains single-stranded nucleic acids. Fully extended primers migrate near the template's full length. (Some variation is observed because XNAs can affect gel mobility by their different mass or hydrodynamic radius.) Longer extension products detected only by SYBR Gold will be the result of template extension.

Blocking the $3^{\prime}$ end of the template with an inverted nucleotide (e.g., $3^{\prime}-3^{\prime} \mathrm{dT}$ ) in synthesis is highly effective at stopping these unwanted extensions. Alternatively, the template can be designed to have a hairpin structure at the $3^{\prime}$ end, acting as both primer and template. In this way, not only are futile secondary extensions abolished but also the efficiency of primer binding to template increases as a consequence of the proximity of the complementary sequences.

\section{Selection of additives}

Co-solutes and crowding agents can have a significant impact on polymerase function, including thermostabilization of the protein and destabilization of the nucleic acid duplex. Commercially available Phi29 DNAP is supplemented with bovine serum albumin (BSA, $0.1 \mathrm{mg} / \mathrm{ml}$ ) for DNA synthesis. We have maintained BSA as an additive in our XNA synthesis reactions, although its effect on the synthesis of nucleic acids other than DNA has not been extensively tested. Of particular interest is the use of polyols like glucose, galactose, trehalose (Pan et al., 2008), mannitol, or sorbitol as they have been reported to both increase Phi29 DNAP thermotolerance and decrease the melting temperature of DNA duplexes, facilitating the strand-displacement activity of the polymerase (K. Davey and V. B. Pinheiro, unpub. data). For the synthesis of HNA polymers, betaine $(1 \mathrm{M})$ has shown to significantly increase the efficiency of the reaction (Figure 3).

\section{Phi29 DNAP purification}

The XNA synthesis activity of a crude $E$. coli lysate of heterologously expressed recombinant Phi29 DNAP is greatly reduced (compared to purified Phi29 DNAP), presumably due to a combination of E. coli proteins present (which may include other polymerases, such as E. coli Pol I) and available priming sites from sheared genomic DNA contamination. Protocols for the efficient purification of Phi29 DNAP free of DNA contaminants have been previously described (Lazaro et al., 1995; Takahashi et al., 2014). Although the quality of the enzyme preparation can be
Torres and Pinheiro 


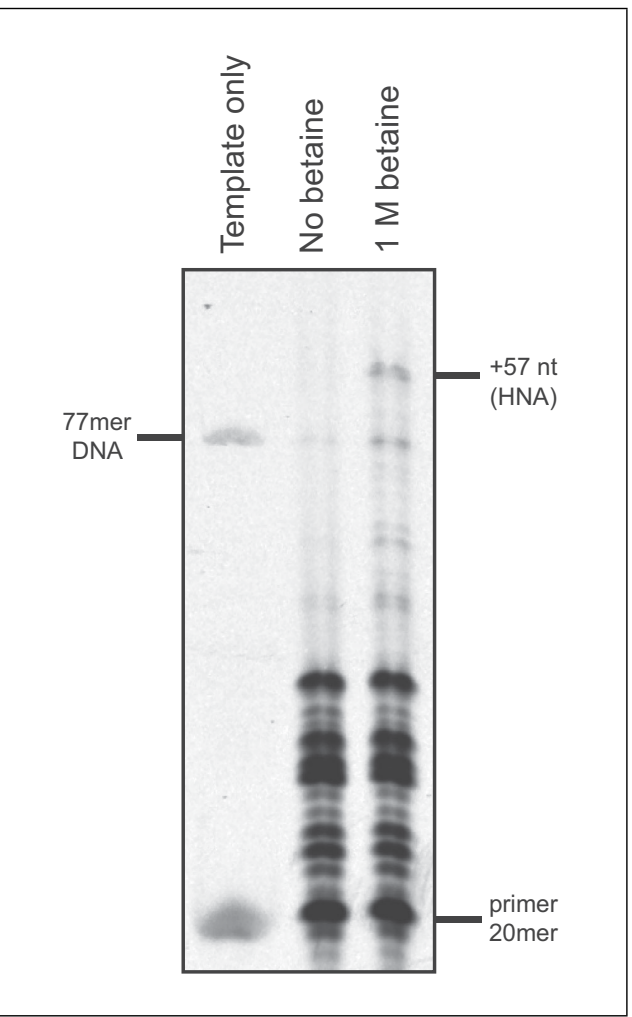

Figure 3 Effect of betaine on Phi29 DNAP HNA synthesis. HNA primer extension products synthesized by Phi29 DNAP N62D without (lane 2) and with (lane 3) $1 \mathrm{M}$ betaine were separated by denaturing PAGE and visualized by gel scanning to detect primer fluorophore signal. Extension conditions were selected to maximize the observable impact of the additive.

crucial for obtaining efficient XNA synthesis, in the particular case of HNA synthesis, partial purification of Phi29 DNAP by heparin adsorption chromatography has proven to be sufficient to obtain a high yield ( $>50 \%)$ of synthesis products of up to 100 incorporations. We typically purify $9 \mu \mathrm{g} \pm 3 \mu \mathrm{g}$ of Phi29 DNA polymerase at $\sim 40 \%$ to $60 \%$ purity from a $50 \mathrm{ml}$ starting culture.

\section{Troubleshooting}

Primer degradation can be a significant issue in XNA synthesis because of the strong exonucleolytic activity of Phi29 DNAP. Primer degradation can have a direct effect on the yield of the XNA synthesis reaction, but in the case of fluorescently labeled primers, primer degradation can also be a source of false positive results when monitoring XNA synthesis by denaturing gel electrophoresis (Figure 4). As primers are degraded, any fluorophore attached to the DNA primer will have a bigger influence on the migration of the oligonucleotide, eventually becoming the dominant

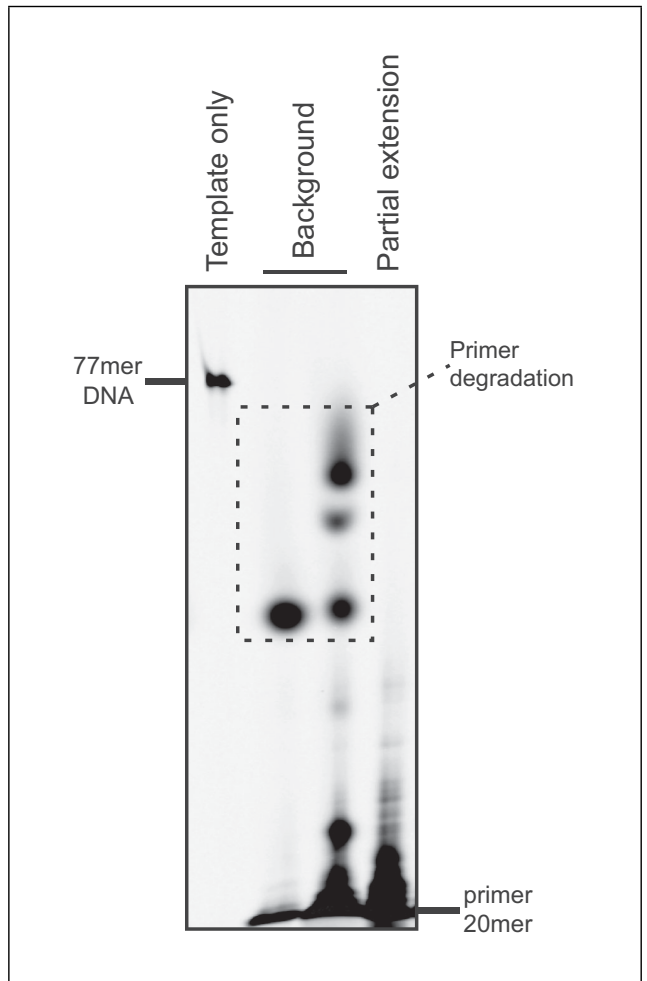

Figure 4 Background signal due to primer degradation. When primer extension reactions are challenging and exonuclease activity is present in the incubation mix, degradation of the primer can take place and background signals, distinctive from extension, can be detected (lanes 2 and 3).

factor in the migration-presumably as the primer is degraded down to a trimer, dimer, or even monomer.

As different dyes have different electrophoretic mobilities, it is possible to rule out the false positive result by using a mixed primer population, each labeled with a different fluorophore (e.g., FAM or IR700). Using primers containing phosphorothioates in their three or four terminal nucleotides ( $3^{\prime}$ end) efficiently blocks primer degradation, which can also be achieved using an exonucleasedeficient Phi29 DNAP, such as D12A presented here.

Multiple factors can lead to reduced XNA synthesis yield and incomplete extensions, particularly Phi29 DNAP activity, XNA triphosphate degradation, and primer-template annealing. A common contaminant in Phi29 purifications from $E$. coli is bacterial DNA and RNA, which can undermine XNA synthesis by providing alternative binding sites for the polymerase. Nucleases like Benzonase (Novagen) or Cyanase (RiboSolutions) efficiently remove nucleic acids from Phi29 DNAP purifications and can be readily removed by 
filtration or resin binding. If this approach is taken, be aware that any contaminating nuclease present in the Phi29 preparation will also interfere with XNA synthesis (by degrading primers and templates).

While protein purity is not essential for Phi29 DNAP activity, XNA synthesis reactions are more robust when Phi29 DNAP is the major component of the purified fraction. Buffer exchange or further purification are effective at removing small molecule or protein contaminants and may allow the polymerase to reach higher concentrations. DNAP concentration can have a major impact on XNA synthesis: Too little enzyme leads to low XNA yields and incomplete extensions, while too much enzyme leads to overextension and template-independent XNA synthesis. Our approach has been to quantify (or estimate) Phi29 DNAP concentration after protein purification and carry out diagnostic experiments that involve titrating the batch of enzyme to identify the range within which XNA synthesis is best, starting from $3 \mathrm{ng}$ DNAP variant per reaction and carrying out 2-fold dilutions to typically $0.1 \mathrm{ng}$ of DNAP per $20 \mu \mathrm{l}$ reaction.

The quality of Phi29 DNAP preparations tend to slowly drop after $-20^{\circ} \mathrm{C}$ storage but generally retain considerable activity for over a month when kept in $1 \times$ Phi29 DNAP storage buffer (New England Biolabs) and 50\% glycerol. Purified DNAP variants can generally be stored at $4^{\circ} \mathrm{C}$ for a week.

XNA triphosphate degradation is possible and may be dependent on the XNA chemistry being used. Stable chemistries such as hNTPs can degrade, whether by inappropriate storage or lower quality purification of the triphosphate stocks. Degradation rarely affects all four XNTP stocks simultaneously and can be readily traced by carrying out XNA synthesis with partial substitutions, where each xNTP is replaced with a natural dNTP (i.e., hATP, hCTP, hGTP, and dTTP to test hA, hC, and hG). For lower XNA synthesis efficiencies, the complementary approach can also be used (i.e., hATP, dCTP, dGTP, and dTTP to test hA only). Notably, triphosphate concentration is an important parameter in XNA synthesis reactions. Above a minimum concentration, which is dependent on the XNA chemistry and polymerase variant being used, XNA synthesis will be carried out efficiently. Phi29 DNAP has high affinity for natural triphosphates $\left(K_{\mathrm{M}} 1 \mu \mathrm{M}\right.$, see Lazaro et al., $1995)$ and for HNA a triphosphate concentration of $50 \mu \mathrm{M}$ is sufficient for the synthesis of short XNA molecules.
Although primer annealing to the template is not commonly an issue, it should also be part of the troubleshooting, particularly for reactions where no XNA appears to be synthesized. Primer-template annealing can be tested using non-denaturing PAGE with primer binding to template detected by a resulting change in mobility.

Finally, reaction parameters and additives (i.e., co-solutes and crowding agents) may also be considered for the optimization of the XNA synthesis, whether to improve low-yield conditions or to curb overextensions. Reaction time can be extended to allow incomplete synthesis to be completed, or shortened to minimize overextension. For DNA synthesis, where the synthesis product is susceptible to exonuclease degradation, we have observed high-yield synthesis as a window: Very short extensions lead to incomplete reactions and overly long extensions (which for DNA would mean $>3 \mathrm{hr}$ for extension of a 100mer template) also result in poor yields due to degradation. For XNAs, overnight extensions generally provide the best results.

The impact of additives is dependent on the XNA chemistry and, in our experience, the effect of these co-solutes is not always additive. For HNA, we have found that betaine has a significant positive effect on XNA synthesis (Figure 3).

\section{Anticipated Results}

Phi29 DNAP-catalyzed HNA synthesis is efficient, and high yields of full-length product should be expected after $1 \mathrm{hr}$ incubation using high-quality enzyme preparations. The electrophoretic mobility of an HNA molecule is lower than a DNA molecule of the same length and sequence, in a polyacrylamide gel, because of the higher mass of hNTPs (Figure 2).

HNA synthesis is dependent on multiple parameters but enzyme concentration and reaction time are the two parameters that most frequently need optimization for synthesis. Purification of synthesized HNA with DNase followed by ethanol precipitation usually recovers $\sim 40 \%$ to $60 \%$ of the starting primer concentration (i.e., 10 pmol HNA from a 20 pmol synthesis scale).

Primer degradation can lead to false positive signals in denaturing PAGE as the mobility of mononucleotides or dinucleotides attached to fluorescent dyes is dominated by the dye, resulting in unusual patterns near the resolving range of the gel. Primer binding to the enzyme can also generate background signals that can
Torres and Pinheiro 
be misinterpreted as XNA synthesis. The presence of $0.1 \%$ SDS in the loading buffer should help to disassemble primer-protein complexes.

\section{Time Considerations}

The recombinant expression, partial purification, and activity test of a batch of Phi29 DNAP requires $\sim 3$ days: One for protein expression, one for protein purification and SDSPAGE confirmation of purity, and a third day to test the DNA activity, titrate the protein, and visualize the results of a primer extension reaction in a denaturing PAGE gel.

For any novel chemistry to be tested, adjusting enzyme concentration, primer-to-template ratio, incubation times, and additive selection can take from 2 to 5 days, depending on how much optimization is necessary.

For HNA, setting up and carrying out a synthesis reaction can be done in $1.5 \mathrm{hr}$. Subsequent separation and visualization of the extension products by urea-PAGE can be completed in $\sim 4 \mathrm{hr}(1.5 \mathrm{hr}$ to set and polymerize the gel, $30 \mathrm{~min}$ to pre-run the gel, $1.5 \mathrm{hr}$ to perform the electrophoresis, and $\sim 20 \mathrm{~min}$ to disassemble and visualize the results).

\section{Acknowledgements}

LT and VBP acknowledge support by the European Research Council [ERC-2013-StG project 336936 (HNAepisome)]. This work was supported by the Biotechnology and Biosciences Research Council (grant number BB/N010221/1).

\section{Literature Cited}

Beese, L. S., \& Steitz, T. A. (1991). Structural basis for the 3'-5 ' exonuclease activity of Escherichia coli DNA polymerase I: A two metal ion mechanism. The EMBO Journal, 10(1), 25-33. doi: 10.1002/j.1460-2075.1991.tb07917.x.

Bernad, A., Blanco, L., Lkaro, J. Y., Martin, G., \& Salas, M. (1989). A Conserved 3' to $5^{\prime}$ exonuclease active site in prokaryotic and eukaryotic DNA polymerases. Cell, 59(1), 219-226. doi: 10.1016/0092-8674(89)90883-0.

Blanco, L., Bernads, A., Lharo, J. M., Martins, G., \& Garmendia, C. (1989). Highly efficient DNA synthesis by the phage Phi29 DNA polymerase. The Journal of Biological Chemistry, 264, 8935-8940.

Blanco, L., \& Salas, M. (1996). Relating structure to function in Phi29 DNA polymerase. Journal of Biological Chemistry, 271(15), 8509-8512. doi: 10.1074/jbc.271.15.8509.

Bramsen, J. B., Grünweller, A., Hartmann, R. K., \& Kjems, J. (2014). Using chemical modification to enhance siRNA performance. In R. K. Hartmann, A. Bindereif, A. Schön, \& Eric Westhof (Eds.), Handbook of RNA biochemistry: Second, completely revised and enlarged edition, (pp. 1243-1278). Weinheim, Germany: WileyVCH. doi: 10.1002/9783527647064.ch56.

Chen, T., Hongdilokkul, N., Liu, Z., Adhikary, R., Tsuen, S. S., \& Romesberg, F. E. (2016). Evolution of thermophilic DNA polymerases for the recognition and amplification of $\mathrm{C} 2^{\prime}$-modified DNA. Nature Chemistry, 8(6), 556-562. doi: 10.1038/nchem.2493.

Cozens, C., Pinheiro, V. B., Vaisman, A., Woodgate, R., \& Holliger, P. (2012). A short adaptive path from DNA to RNA polymerases. Proceedings of the National Academy of Sciences of the United States of America, 109(21), 8067-8072. doi: 10.1073/pnas.1120964109.

Culbertson, M. C., Temburnikar, K. W., Sau, S. P., Liao, J.-Y., Bala, S., \& Chaput, J. C. (2016). Evaluating TNA stability under simulated physiological conditions. Bioorganic \& Medicinal Chemistry Letters, 26(10), 2418-2421. doi: 10.1016/j.bmcl.2016.03. 118.

Dean, F. B., Nelson, J. R., Giesler, T. L., \& Lasken, R. S. (2001). Rapid amplification of plasmid and phage DNA using Phi 29 DNA polymerase and multiply-primed rolling circle amplification. Genome Research, 11(6), 1095-1099. doi: 10.1101/gr.180501.

Di Pasquale, F., Fischer, D., Grohmann, D., Restle, T., Geyer, A., \& Marx, A. (2008). Opposed steric constraints in human DNA polymerase $\beta$ and E. coli DNA polymerase I. Journal of the American Chemical Society, 130(32), 10748-10757. doi: $10.1021 / \mathrm{ja} 8028284$.

Dunn, M. R., Otto, C., Fenton, K. E., \& Chaput, J. C. (2016). Improving polymerase activity with unnatural substrates by sampling mutations in homologous protein architectures. ACS Chemical Biology, 11(5), 1210-1219. doi: 10.1021/acschembio.5b00949.

Ellington, A., \& Pollard, J. D. (1998). Purification of oligonucleotides using denaturing polyacrylamide gel electrophoresis. Current Protocols in Molecular Biology, 2.12.1-2.12.7. doi: 10.1002/0471142727.mb0212s42.

Esteban, J. A., Salas, M., \& Blanco, L. (1993). Fidelity of phi29 DNA polymerase. Comparison between protein-primed initiation and DNA polymerization. The Journal of Biological Chemistry, 268(4), 2719-2726.

Esteban, J. A., Soengas, M. S., Salas, M., \& Blanco, L. (1994). 3' $\rightarrow 5^{\prime}$ exonuclease active site of Phi29 DNA polymerase. Evidence favoring a metal ion-assisted reaction mechanism. The Journal of Biological Chemistry, 269(50), 31946-31954.

Gardner, A. F., Joyce, C. M., \& Jack, W. E. (2004). Comparative kinetics of nucleotide analog incorporation by vent DNA polymerase. The Journal of Biological Chemistry, 279(12), 1183411842. doi: 10.1074/jbc.M308286200.

Garmendia, C., Bernad, A., Esteban, J. A., Blanco, L., \& Salas, M. (1992). The bacteriophage Phi29 DNA polymerase, a proofreading enzyme. The Journal of Biological Chemistry, 267(4), 25942599. 
Ghadessy, F. J., Ramsay, N., Boudsocq, F., Loakes, D., Brown, A., Iwai, S., . . Holliger, P. (2004). Generic expansion of the substrate spectrum of a DNA polymerase by directed evolution. Nature Biotechnology, 22(6), 755-759. doi: 10.1038/nbt974.

Ibach, J., Dietrich, L., Koopmans, K. R. M., Nobel, N., Skoupi, M., \& Brakmann, S. (2013). Identification of a T7 RNA polymerase variant that permits the enzymatic synthesis of fully 2'-O-methyl-modified RNA. Journal of Biotechnology, 167(3), 287-295. doi: 10.1016/j.jbiotec.2013.07.005.

Ichida, J. K., Horhota, A., Zou, K., McLaughlin, L. W., \& Szostak, J. W. (2005). High fidelity TNA synthesis by Therminator polymerase. $\mathrm{Nu}$ cleic Acids Research, 33(16), 5219-5225. doi: 10.1093/nar/gki840.

Lagoja, I., Marchand, A., Van Aerschot, A., \& Herdewijn, P. (2003). Synthesis of 1,5anhydrohexitol building blocks for oligonucleotide synthesis. Current Protocols in $\mathrm{Nu}$ cleic Acid Chemistry, 14, 1.9:1.9.1-1.9.22. doi: 10.1002/0471142700.nc0109s14.

Lazaro, J. M., Blanco, L., \& Salas, M. (1995). Purification of bacteriophage $\varphi 29$ DNA polymerase. Methods in Enzymology, 262(1984), 42-49. doi: 10.1016/0076-6879(95)62007-9.

Mönttinen, H. A. M., Ravantti, J. J., Stuart, D. I., \& Poranen, M. M. (2014). Automated structural comparisons clarify the phylogeny of the right-hand-shaped polymerases. Molecular Biology and Evolution, 31(10), 2741-2752. doi: 10.1093/molbev/msu219.

Pan, X., Urban, A. E., Palejev, D., Schulz, V., Grubert, F., Hu, Y., ... Weissman, S. M. (2008). A procedure for highly specific, sensitive, and unbiased whole-genome amplification. Proceedings of the National Academy of Sciences of the United States of America, 105(40), 1549915504. doi: 10.1073/pnas.0808028105.

Pinheiro, V. B., \& Holliger, P. (2014). Towards XNA nanotechnology: New materials from synthetic genetic polymers. Trends in Biotechnology, 32(6), 321-328. doi: 10.1016/ j.tibtech.2014.03.010.

Pinheiro, V. B., Loakes, D., \& Holliger, P. (2012). Synthetic polymers and their potential as genetic materials. Bioessays, 35(2), 113-122. doi: 10.1002/bies.201200135.

Ramsay, N., Jemth, A.-S., Brown, A., Crampton, N., Dear, P., \& Holliger, P. (2010). CyDNA: Synthesis and replication of highly Cy-dye substituted DNA by an evolved polymerase. Journal of the American Chemical Society, 132(14), 5096-5104. doi: 10.1021/ja909180c.

Rodríguez, I., Lázaro, J. M., Blanco, L., Kamtekar, S., Berman, A. J., Wang, J., ... de Vega, M. (2005). A specific subdomain in $\varphi 29$ DNA polymerase confers both processivity and stranddisplacement capacity. Proceedings of the $\mathrm{Na}$ tional Academy of Sciences of the United States of America, 102(18), 6407-6412. doi: 10.1073/pnas.0500597102.
Ruckman, J., Green, L. S., Beeson, J., Waugh, S., Gillette, W. L., Henninger, D. D., ... Janjić, N. (1998). 2'-Fluoropyrimidine RNA-based aptamers to the 165-amino acid form of vascular endothelial growth factor (VEGF165). The Journal of Biological Chemistry, 273(32), 2055620567. doi: 10.1074/jbc.273.32.20556.

Salas, M. (1991). Protein-priming Of DNA replication. Annual Review of Biochemistry, 60, 39-71. doi: 10.1146/annurev.bi.60.070191.000351.

Salas, M., Holguera, I., Redrejo-Rodríguez, M., \& de Vega, M. (2016). DNA-binding proteins essential for protein-primed bacteriophage $\Phi 29$ DNA replication. Frontiers in Molecular Biosciences, 3, 1-21. doi: 10.3389/ fmolb.2016.00037.

Soengas, M. S., Esteban, J. A., Lazaro, J. M., Bernad, A., Blasco, M. A., Salas, M., \& Blanco, L. (1992). Site-directed mutagenesis at the Exo Ill motif of phi 29 DNA polymerase; overlapping structural domains for the $3^{\prime}-5^{\prime}$ exonuclease and strand-displacement activities. The EMBO Journal, 11, 4227-4237.

Soengas, M. S., Gutiérrez, C., \& Salas, M. (1995). Helix-destabilizing activity of phi 29 single-stranded DNA binding protein: Effect on the elongation rate during strand displacement DNA replication. Journal of Molecular Biology, 253(4), 517-529. doi: 10.1006/jmbi.1995.0570.

Sousa, R., \& Padilla, R. (1995). A mutant T7 RNA polymerase as a DNA polymerase. The EMBO Journal, 14(18), 4609-4621.

Summer, H., Grämer, R., \& Dröge, P. (2009). Denaturing urea polyacrylamide gel electrophoresis (Urea PAGE). Journal of Visualized Experiments, 32, 3-5. doi: 10.3791/1485.

Takahashi, H., Yamazaki, H., Akanuma, S., Kanahara, H., Saito, T., Chimuro, T., ... Kobori, T. (2014). Preparation of Phi29 DNA polymerase free of amplifiable DNA using ethidium monoazide, an ultraviolet-free light-emitting diode lamp and trehalose. PLoS One, 9(2), e82624. doi: 10.1371/journal.pone.0082624.

Taylor, A. I., \& Holliger, P. (2015). Directed evolution of artificial enzymes (XNAzymes) from diverse repertoires of synthetic genetic polymers. Nature Protocols, 10(10), 1625-1642. doi: 10.1038/nprot.2015.104.

Taylor, A. I., Beuron, F., Peak-Chew, S. Y., Morris, E. P., Herdewijn, P., \& Holliger, P. (2016). Nanostructures from synthetic genetic polymers. ChemBioChem, 17, 1107-1110. doi: 10.1002/cbic.201600136.

Vega, M. De, Lazaro, J. M., Salas, M., \& Blanco, L. (1996). Primer-terminus stabilization at the 3'-5' exonuclease active site of phi29 DNA polymerase. Involvement of two amino acid residues highly conserved in proofreading DNA polymerases. The EMBO Journal, 15(5), 11821192.

Xiong, S., Zhang, L., \& He, Q. Y. (2008). Fractionation of proteins by heparin chromatography. Methods in Molecular Biology, 424, 213-221. doi: 10.1007/978-1-60327-064-9_18.
Torres and Pinheiro 